\title{
Can We Observe the Gravitational Quantum States of Positronium?
}

\author{
P. Crivelli, ${ }^{1}$ V. V. Nesvizhevsky, ${ }^{2}$ and A. Yu. Voronin ${ }^{3}$ \\ ${ }^{1}$ ETH Zurich, Institute for Particle Physics, Otto-Stern-Weg 5, 8093 Zurich, Switzerland \\ ${ }^{2}$ Institut Laue-Langevin, 6 rue Jules Horowitz, 38046 Grenoble, France \\ ${ }^{3}$ Lebedev Physical Institute, 53 Leninsky Prospect, Moscow 119991, Russia
}

Correspondence should be addressed to P. Crivelli; paolo.crivelli@cern.ch

Received 6 June 2014; Revised 17 August 2014; Accepted 29 August 2014

Academic Editor: Stefan Baessler

Copyright (C) 2015 P. Crivelli et al. This is an open access article distributed under the Creative Commons Attribution License, which permits unrestricted use, distribution, and reproduction in any medium, provided the original work is properly cited. The publication of this article was funded by SCOAP S $^{3}$

\begin{abstract}
We consider the feasibility of observing the gravitational quantum states of positronium. The proposed scheme employs the flow-throw technique used for the first observation of this effect with neutrons. Collimation and Stark deceleration of Rydberg positronium atoms allow selecting the required velocity class. If this experiment could be realized with positronium, it would lead to a determination of $g$ for this matter-antimatter system at the few \% level. As discussed in this contribution, most of the required techniques are currently available but important milestones have to be demonstrated experimentally before such an experiment could become reality. Those are the efficient focusing of a bunched positron beam, Stark deceleration of Rydberg positronium, and its subsequent excitation into states with large angular momentum. We provide an estimate of the efficiencies we expect for these steps and assuming those could be confirmed we calculate the signal rate.
\end{abstract}

\section{Introduction}

Quantum gravitational states were observed for the first time with neutrons by measuring their transmission through a slit made of a mirror and an absorber [1]. If the distance between the mirror and the absorber (which is a rough surface used as a scatterer to mix the velocity components) is much higher than the turning point for the corresponding gravitational quantum state, the neutrons pass through the slit without significant losses. As the slit size decreases the absorber starts approaching the size of the neutron wave function and the probability of neutron loss increases. If the slit size is smaller than the characteristic size of the neutron wave function in the lowest quantum state, the slit is not transparent for neutrons as this was demonstrated experimentally. The height and energies of the gravitational quantum states can be determined analytically and the solution of the Schrödinger equation contains airy functions. A more transparent and simple equation can be derived using a semiclassical approach [2]. This solution reproduces the energy of the gravitational states within $1 \%$ and is given by

$$
E_{n} \simeq \sqrt[3]{\frac{9 m}{8} \cdot\left(\pi \hbar g\left(n-\frac{1}{4}\right)\right)},
$$

where $m$ is the particle mass, $g$ the gravitational acceleration, $n$ the principal quantum number, and $\hbar$ the reduced Planck constant. The characteristic scale for the gravitational quantum states is equal to

$$
z_{0}=\sqrt[3]{\frac{\hbar^{2}}{2 m^{2} g}} .
$$
by

The corresponding classically allowed heights are given

$$
z_{n}=\frac{E_{n}}{m g}=\lambda_{n} z_{0},
$$

where $\lambda_{n}=\{2.34,4.09,5.52,6.79,7.94,9.02,10.04, \ldots\}$ are the zeros of the airy function. For neutrons the height of the lowest gravitational level is $13.7 \mu \mathrm{m}$. For positronium, the electron-positron bound state, that is, 1000 times lighter than 


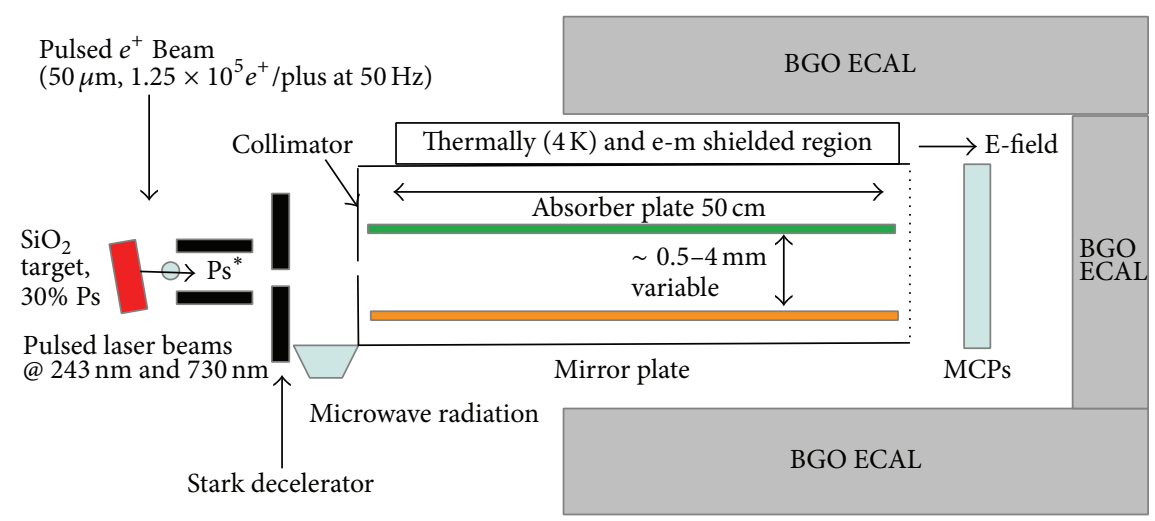

FIGURE 1: Scheme of the proposed experimental setup.

a neutron, one gets a 100 larger size corresponding to $z_{1}=$ $1.3 \mathrm{~mm}$ while the energy is 10 times smaller, $E_{1}=0.13 \mathrm{peV}$. The observation time to resolve a quantum gravitational state can be estimated using the Heisenberg uncertainty principle to be of the order of $\hbar / E_{1} \simeq 4.5 \mathrm{~ms}$. This value is much larger than the long lived triplet positronium lifetime in the ground state which is 142 ns (the Ps singlet state lives only 125 ps and thus in the following we will only consider the triplet state and refer to it as Ps). Luckily, the Ps lifetime can be increased by exciting it to a higher level. In a Rydberg state the Ps lifetime against annihilation is increased by a factor of $n^{3}$, where $n$ is the principal quantum number, because of the decrease of the overlap of the positron and the electron wave functions. As for the case of a measurement of the gravitational free fall of Ps proposed by Mills and Leventhal [3], the usable lifetime to observe a quantum gravitation state of a Rydberg Ps atom (hereafter Ps ${ }^{*}$ ) is the one before it emits the first photon. In fact, after that the recoil will modify its trajectory and vertical energy; thus the Ps atom will be lost inside the slit. For this reason, the excited Ps has to be spun up to high $l$ values with circularly polarized microwave radiation.

\section{Experimental Technique}

A scheme of the proposed experimental setup is shown in Figure 1. Positronium is formed by implanting keV positrons from a remoderated pulsed slow positron beam in a positronpositronium converter (see Section 3.3). To observe the quantum mechanical behavior of Ps in the gravitational field its vertical velocity should be of the same order as the gravitational energy levels and thus $v_{y}<0.15 \mathrm{~m} / \mathrm{s}$. Furthermore to resolve the quantum state the Ps atom has to interact long enough with the slit and therefore it has to be laser excited to a Rydberg state with $n>30$ and maximum $l$ quantum number. To keep a reasonable size of the experimental setup (i.e., a slit size of the order of $0.5 \mathrm{~m}$ ) and minimize the number of detectors the velocities in the horizontal plane should be smaller than $v_{x, z}<100 \mathrm{~m} / \mathrm{s}$. Similar to neutrons a collimator will be used to select the velocity components $v_{x}, v_{y}$ of the positronium distribution. However since no reliable thermal cold source of positronium exists, the velocity component perpendicular to the surface $v_{z}$ has to be lowered by some other means. Relying to the fact that atoms in Rydberg states have a large dipole moment, Stark deceleration can be used for this purpose. After deceleration the $\mathrm{Ps}^{*}$ are driven by circularly polarized microwaves to a state with high $l$. If the slit width is smaller than the first expected gravitational state (i.e., $<1 \mathrm{~mm}$ ), this will not be transparent and therefore no signal will be detected above the expected background in the detectors. If the width is increased to a value lying between the first and the second gravitational state (i.e., $<2 \mathrm{~mm}$ ) the Ps wavefunction can propagate and a signal is expected to be detected. This quantum jump provides the unambiguous indication of the observation of a quantum gravitational state of positronium.

\section{Experimental Setup}

3.1. Positron Beam. Few facilities around the world are nowadays capable of producing fluxes of positrons exceeding $1 \times 10^{8} e^{+} / \mathrm{s}$ [4-11]. The highest intensity reported so far has been reached at the FMR II NEMOPUC source in Munich where a monoenergetic slow positron beam of $9 \times 10^{8} e^{+} / \mathrm{s}$ has been achieved [12]. We will base our estimation of the signal rate assuming that such a beam could be used. In principle, even higher fluxes are conceivable (see, e.g., $[13,14])$ and hopefully those will be available in the near future.

The beam will be coupled to a buffer gas trap. This is necessary in order to produce positron pulses at a rate of tens of $\mathrm{Hz}$ to synchronize positronium formation with pulsed lasers in order to drive the transition of Ps in a Rydberg state. The typical trapping efficiency is $20 \%$ and the positron lifetime in the trap is $2 \mathrm{~s}$. Therefore above a few $\mathrm{Hz}$ repetition rate, the losses are minimized. The positrons are dumped in bunches of $20 \mathrm{~ns}$ using the standard electrodes of the trap and by adding a buncher one can achieve $<1 \mathrm{~ns}$ pulses $[15,16]$. The typical positron beam size of such a system is $1 \mathrm{~mm}$ (FWHM). This has to be reduced via positron remoderation in order to achieve a good geometrical overlap of the Ps source with the opening of the slit (mirror-absorber).

Note that a new concept for a positron buffer gas trap with the prospect of obtaining up to $92 \%$ trapping efficiencies has been recently proposed [17].

3.2. Remoderation. Remoderation of positron beam allows brightness enhancement. Microbeams of 1-2 $\mu \mathrm{m}$ were developed by the use of two successive stages of focusing, acceleration, and remoderation [18]. The loss of beam intensity is 
about a factor 10 per stage in reflection geometry. To achieve a positron beam of $50 \mu \mathrm{m}$, only one moderator stage will be required. One can use a $\mathrm{Ni}(100)$ foil in transmission geometry which even being a factor 2 less efficient allows the beam to be extracted from the magnetic field region with a transmission of $71 \%[19,20]$. Such a remoderator leads to about $5 \%$ of the incoming positrons in a beam of $50 \mu \mathrm{m}[19,20]$. This was achieved using a continuous positron beam but it has not been yet demonstrated for a bunched beam. Such a scheme is currently under investigation at the University College of London (UCL) for their ongoing free fall measurement of Ps. Their preliminary simulations show that one should be able to obtain $1 \mathrm{~ns}$ positron bunches of about $25 \mu \mathrm{m}$ spot size [21]. Assuming that a remoderation efficiency of $5 \%$ could be attained, the positron flux at the target will be of the order of $6.3 \times 10^{6} e^{+} / \mathrm{s}\left(1.25 \times 10^{5} e^{+}\right.$per bunch at a repetition rate of $50 \mathrm{~Hz})$.

3.3. Positronium Source. Positrons implanted in silica thin films with $3 \mathrm{keV}$ will form Ps emitted into vacuum with an efficiency of about $30 \%$ and an energy of $75 \mathrm{meV}[22,23]$. The vacuum yield is basically constant in the temperature range from $50 \mathrm{~K}$ to $400 \mathrm{~K}$ [22]. The emission energy of Ps is determined by the diameter of the pores $(3 \mathrm{~nm})$. In fact for Ps energies of a few hundred meV, the Ps de Broglie wavelength is comparable with the energy levels of positronium confined inside a pore of this size and therefore it has to be treated quantum mechanically. A simple particle in a box model is a good approximation to describe Ps in such porous materials $[22,24,25]$. Ps formed inside the film with an initial energy of $1 \mathrm{eV}$ (corresponding to the negative workfunction of Ps in silica) starts diffusing through the interconnected pore network tunneling from one pore to the other and loses energy via collision until the ground state energy is attained. A fraction of those atoms (30\% of the incoming positrons) will make it to the surface before decaying and are emitted into vacuum. At temperatures below $100 \mathrm{~K}$ in films of 3$4 \mathrm{~nm}$ diameter only the ground state of Ps in the pore is populated. Therefore, Ps emitted into vacuum is basically monoenergetic. Since the pore orientation at the surface of these materials is random, the emission distribution follows a $\cos \theta$ distribution [22, 23].

At $3 \mathrm{keV}$, the positrons have a mean implantation depth of $150 \mathrm{~nm}$. The time required for the Ps atoms to exit the film and be emitted into vacuum has been measured to be about $1 \mathrm{~ns}$ [26]. Since the areal density per pulse is of the order of $10^{9} \mathrm{Ps} / \mathrm{cm}^{2}$, the spin exchange quenching and formation of molecular positronium inside the films should be considered. The total cross section in those targets for these processes has been determined to be $3.4 \times 10^{-14} \mathrm{~cm}^{-2}$ [27]. In those measurements, a source based positron beam was used and at areal densities of $4 \times 10^{10} \mathrm{Ps} / \mathrm{cm}^{2}$ a saturation of the quenching process was observed. A fraction of 0.3 of the initial positronium population survives since the positrons from a radioactive source are spin polarized (due to parity violation). For positrons produced in a reactor, this is not the case and therefore the losses will be higher. However, using the measured cross sections and taking into account that once emitted into vacuum after $1 \mathrm{~ns}$ the Ps cloud expands and therefore the density decreases rapidly, we estimated that this effect can be neglected.

To reduce the perpendicular velocity $v_{z} \simeq 10^{5} \mathrm{~m} / \mathrm{s}$, we plan to use Stark deceleration. This method has been applied to different atomic species (including hydrogen) [28] and molecules [29]. Atoms in Rydberg states have large dipole moments; thus electric field gradients can be used to manipulate them. The acceleration/deceleration $a$ imparted to the Rydberg atoms is given by

$$
a=76 \nabla F \frac{1}{m} n k,
$$

where $\nabla F$ is the gradient of the electric field in $\mathrm{Vcm}^{-2}, m$ the mass of the decelerated particles in atomic units, and $n$ and $k$ the Stark state quantum numbers. $\mathrm{H}$ atoms in $n=25$ and an initial velocity of $700 \mathrm{~m} / \mathrm{s}$ can be brought at rest in $3 \mathrm{~mm}$ [28]. For Ps being 1000 times lighter, decelerations exceeding $10^{9} \mathrm{~m} / \mathrm{s}$ could be realized. Since one is interested only in decelerating the distribution that is almost perpendicular to the surface of the Ps target, one can expect an efficiency close to $100 \%$. This is confirmed by preliminary simulations (private communication with Dr. C. Seiler). The collimator will be placed after the deceleration stage and the microwave region where circularly polarized radiation will spin up the Ps to the maximum $l$ so that kicks to the momentum imparted to the atoms in the vertical direction during these processes will be accounted for.

The fraction of atoms with $v_{y}<0.15, v_{x}, v_{z}<100 \mathrm{~m} / \mathrm{s}$ is estimated using a Monte Carlo simulation taking into account the angular of the Ps emitted into vacuum from porous silica to be of the order of $2 \times 10^{-9}$.

Other promising candidates for Ps formation that should be considered for this experiment are aluminum oxidized nanochannels of 5-8 nm [30], Si and Ge surfaces [31], and hydrophobic silica aerogels [32].

To produce a source of cold Ps, another option would be to use the tails of the distribution of Ps thermally desorbed and emitted into vacuum. For $\mathrm{Al}(111)$ kept at $600 \mathrm{~K}$, the conversion efficiency $e^{+}$-Ps was measured to be as high as $30 \%$ [33]. However, the surface quality is very important, and degradation is observed in a short time scale especially when a laser is positioned near the surface [31,34]. Therefore, until stable desorption at room temperature (or lower) could be demonstrated, this does not seem to be an attractive path.

3.4. Laser for Rydberg Excitation. Positronium excitation in Rydberg states was first demonstrated by Ziock et al. [35]. Recently, an excitation of Rydberg Ps with a two-step process $1 \mathrm{~S} \rightarrow 2 \mathrm{P}$ and $2 \mathrm{P} \rightarrow 25$ with an efficiency of $25 \%$ was reported $[36,37]$. Two broadband $(150 \mathrm{GHz})$ dye lasers were used in order to maximize the overlap with the large Doppler profile of the Ps atoms. This led to an occupation of the Stark levels that could not be resolved. In the experiment proposed here, one is interested to excite only a small fraction of the atoms with the correct velocity characteristic. The lasers axis will be set parallel to the $v_{x}$ component. For $v_{x}=100 \mathrm{~m} / \mathrm{s}$, the Doppler profile will be of the order of $1 \mathrm{GHz}$ and thus 
narrow dye lasers at $243 \mathrm{~nm}$ and $730 \mathrm{~nm}$ can be used. One has to consider that in both cases the photon absorbed by the atom will result in recoil and therefore the laser frequency has to be tuned in order to compensate for this effect. This can be optimized by detecting the Rydberg atoms via field ionization after the collimator slit maximizing their flux. In such a scheme, the excitation probability will be at least as large as $25 \%$ (as for the case of broadband excitation) with the advantage of populating only the desired Stark state $(n=33$ and $k=19)$. As a base for our estimation of the signal rate, we take conservatively this value. An alternative might be to use Doppler free two-photon excitation from the ground state directly to $n=33$. This could lead to higher efficiencies exceeding $50 \%[21,38]$ but it has not been yet realized for Ps. After deceleration, the Ps ${ }^{*}$ will be driven to its highest circular state $l=32$ using polarized microwave radiation.

3.5. Mirror and Absorber. As a mirror for Ps, we propose to exploit a gradient of magnetic field created using wires arranged parallel to each other with a constant current to create a uniform gradient of the magnetic field. Only the Ps triplet atoms with $m=0$ have a nonzero net magnetic moment. For the $m= \pm 1$, the electron and the positron magnetic moments cancel and therefore those are insensitive to the magnetic field. Therefore, only one-third of the initial population will be reflected. To equate the $E_{y}=0.1 \mathrm{peV}$, a field of a few $\mathrm{mG}$ at the wire surface will be sufficient.

Because of the large spatial size of gravitational quantum states and the very large characteristic length of the mirror needed to form the gravitational states that is much larger than a characteristic interwire distance, we expect that the very weak magnetic gradient will not perturb the gravitational states. The strict theoretical analysis of this clearly mathematically defined problem is ongoing.

It is important to note that a well-defined $(n, l)$ state should be used in order to avoid the fact that the spread in the magnetic moment would wash out the transmission versus the slit height dependence.

A matter mirror should also be considered. Due to the large spatial size of the gravitational quantum state, the surface potential is expected to be very sharp and therefore result in efficient quantum reflection [39]. In both cases (magnetic or material mirror), we expect to have effectively (quasiclassically) only a few collisions with surface. Nevertheless, the transitions rates due to quenching and ionization caused by the electric or magnetic fields have to be calculated.

The absorber as for the neutrons is a rough surface on which the impinging Ps will mix its velocity components and therefore be lost (i.e., it will not be reflected by the mirror).

3.6. Detectors. The detectors are made of an array of ten standard rectangular MCPs (e.g., Hamamatsu F4772-01, $55 \times$ $8 \mathrm{~mm}^{2}$ ) placed $50 \mathrm{~mm}$ from the end of the mirror-absorber region. A uniform electric field will ionize the Ps* and guide the positrons to the MCPs where they can be detected with high efficiency $(80 \%)$. In order to reduce accidental background, the MCPs will be surrounded by an almost $4 \pi$ calorimeter made of 100 BGOs (hexagonal shape of $55 \mathrm{~mm}$ outer diameter and $200 \mathrm{~mm}$ length) placed outside of the vacuum chamber to detect the back to back $511 \mathrm{keV}$ annihilation gamma rays from the $e^{+}$annihilation. This detector was used in the search for Ps $\rightarrow$ invisible decays where the efficiency for detection of two $511 \mathrm{keV}$ photons was larger than 99.99999\% [40]. An energy cut of $400 \mathrm{keV}<E<600 \mathrm{keV}$ will be applied and the coincidence between the two back to back crystals within $10 \mathrm{~ns}$ will be required. Based on simulation with Geant 4 (validated with measurements that used a BGO calorimeter), this will result in an efficiency for the detection of the photons from the positrons annihilation of more than $80 \%$ (arising from the geometrical limitations that do not allow for a full coverage). The measured background for such a signature for the BGO detectors is of the order of 0.25 events/s. By adding the requirement that a positron is detected in the MCP (dark count of 100 counts/s) within 10 ns will result in 0.04 background event/day accidental rate (this includes environmental, internal BGO radioactivity, and cosmic rays). The combination of the coincidence between the positron detection in the MCP and the detection of its annihilation photons will result in an efficiency of $64 \%$. The positronium time-of-flight will be used to suppress accidentals from the prompt positron and positronium annihilation.

3.7. Experimental Environment Considerations. Due to their sensitivity to black body radiation, the absorber and the mirror have to be kept at $4 \mathrm{~K}$. Furthermore, special care has to be taken in order to avoid stray electric and magnetic fields. A mumetal shielding should be foreseen. The target and the electrodes for the Rydberg deceleration can be kept at higher temperature of $150 \mathrm{~K}$ since the collimator will act as a thermal shielding and in addition it will prevent a leak of the electric fields from the deceleration stage. The MCPs are also kept at this higher temperature.

\section{Expected Rate}

The expected signal rate can be estimated using

$$
\begin{aligned}
R_{\text {signal }} & =R_{e+} \cdot \epsilon_{\mathrm{Ps}} \cdot \epsilon_{n 33} \cdot \epsilon_{l 32} \cdot \epsilon_{\text {collimator }} \cdot \epsilon_{\mathrm{tau}} \cdot \epsilon_{\mathrm{dec}} \cdot \epsilon_{\mathrm{det}} \\
& =8.7 \times 10^{-6} \text { events } / \mathrm{s},
\end{aligned}
$$

where

(i) $R_{e+}=6.25 \times 10^{6} e^{+} / \mathrm{s}$ is the positron flux on target. This was estimated assuming that one could use the full intensity of the currently strongest existing positron source, a buffer gas trap efficiency of $20 \%$, extraction losses to a field free magnetic field region of $70 \%$, and a remoderation efficiency of $5 \%$. This last one was realized for a continuous positron beam but not yet for a bunched beam as required for this experiment (see Section 3.2);

(ii) $\epsilon_{\mathrm{Ps}}=0.1$ is the fraction of Ps emitted into vacuum from the proposed porous silica film. This includes the losses due to spin quenching and $\mathrm{Ps}_{2}$ molecule formation and the fact that only the $m=0$ triplet state will be reflected from the proposed mirror; 
(iii) $\epsilon_{n 33}=0.25$ is the excitation probability in the $n=33$, $k=19$ state;

(iv) $\epsilon_{l 32}=0.1$ is the efficiency to drive the Ps in the $n=33$, $l=32$ state. This has been realized for other atomic species (see, e.g., [41] and reference therein) but never for Ps;

(v) $\epsilon_{\text {collimator }}=2 \times 10^{-9}$ is the fraction of Ps atoms remaining after the collimator $\left(v_{x}<100 \mathrm{~m} / \mathrm{s}\right.$ and $v_{y}<0.15 \mathrm{~m} / \mathrm{s}$ ). This was estimated with a Monte Carlo simulation (see Section 3.3);

(vi) $\epsilon_{\mathrm{tau}}=0.5$ is the fraction of atoms in the $n=33, l=32$ state, which has a lifetime of about $8 \mathrm{~ms}$, surviving the $5 \mathrm{~ms}$ time-of-flight;

(vii) $\epsilon_{\mathrm{dec}}=0.8$ is the deceleration efficiency for the $v_{z}<100 \mathrm{~m} / \mathrm{s}$ estimated with preliminary simulations validated for different atomic species;

(viii) $\epsilon_{\mathrm{det}}=0.64$ is the efficiency of signal detection.

Assuming that all the efficiencies quoted above could be confirmed experimentally, a rate of 0.7 events/day with a background 0.05 events/day is anticipated. Possible losses due to spurious effects like stray electric or magnetic fields or black body radiation seem to be negligible but further calculations and preliminary experiments should be done to confirm this assumption.

Note that the expected height of the gravitational state is related to $g$ by (3). This means that for an uncertainty in the determination of $z_{1}$ of $d z_{1}$ one can get an accuracy in the determination of $g$ at the level of $d g / g=3 d z_{1} / z_{1} \sqrt{N}$, where $N$ is the number of detected signals. Assuming an uncertainty of $d z_{1}=0.1 \mathrm{~mm}$ which is mainly determined by the finite source size, the value of $d g / g$ can be determined to $3 \%$ in three months. This is comparable to the accuracy that is aimed for by the antihydrogen experiments at CERN [42-44]. Therefore, observation of Ps gravitational quantum states offers a complementary approach to test the effect of gravity on a pure leptonic system. In comparison to a freefall experiment [21], perturbations of the Ps atoms arising from uncontrolled patch electric fields will not result in a systematic effect for the experiment but will only affect the signal rate.

\section{Conclusions}

The observation of the gravitational quantum states of positronium is very challenging and at this point we cannot conclude that it is feasible. In fact, even though most of the techniques are currently available, some essential milestones have to be demonstrated experimentally before such an experiment could be realized. Those are the efficient focusing of a bunched positron beam, the Stark deceleration of Rydberg positronium, and the subsequent excitation of Rydberg positronium into states with large angular momentum. In this contribution, we provide our expectations for the efficiencies of these various steps based on published work with Ps and other atomic species. Our estimation of the signal rate is encouraging thus justifying and stimulating preliminary experiments, further calculations, and simulations. Furthermore, the developed techniques could also find an application in other fields. Rydberg deceleration of Ps atoms would be a major step to open a new era in Ps spectroscopy. If this would be realized, the main systematical effects (second order Doppler shift and time-of-flight broadening) would be reduced by two orders of magnitude (well below its natural linewidth of $1.2 \mathrm{MHz}$ ); thus an uncertainty in the measurement of the $1 S-2 S$ interval of positronium at the few $\mathrm{kHz}$ level might be possible. Assuming that the QED corrections could be calculated at the same level, this would lead to a new, independent (Ps, being purely leptonic, is free of nuclear size effects) determination of the Rydberg constant at the same level of precision as the current one [45]. This might help to shed some light on the proton size puzzle $[46,47]$ since this can be solved by shifting by 5 standard deviations the value of the Rydberg constant [48].

If an observation of the gravitational quantum states of positronium could be realized, this would result in a determination of $\mathrm{dg} / \mathrm{g}$ for positronium at a level of $3 \%$ assuming 70 events could be detected. If successful, the ongoing efforts to increase the positron buffer gas trap efficiency [17], the available slow positron fluxes, seeking to produce a source of colder Ps [30-32], would certainly help to make the observation of the gravitational quantum states of positronium feasible.

\section{Conflict of Interests}

The authors declare that there is no conflict of interests regarding the publication of this paper.

\section{Acknowledgments}

The authors wish to thank the GRANIT collaboration for providing excellent possibilities for discussions and exchange. P. Crivelli acknowledges the support by the Swiss National Science Foundation (Grant PZ00P2 132059) and ETH Zurich (Grant ETH-47-12-1) and the very useful discussions with F. Merkt, C. Seiler, D. A. Cooke, and D. J. Murtagh.

\section{References}

[1] V. V. Nesvizhevsky, H. G. Börner, A. K. Petukhov et al., "Quantum states of neutrons in the Earth's gravitational field," Nature, vol. 415, no. 6869, pp. 297-299, 2002.

[2] L. D. Landau and E. M. Lifshitz, Quantum Mechanics, Pergamon Press, Oxford, UK, 1976.

[3] A. Mills and M. Leventhal, "Can we measure the gravitational free fall of cold Rydberg state positronium?” Nuclear Instruments and Methods B, vol. 192, pp. 102-106, 2002.

[4] A. van Veen, H. Schut, F. Labohm, and J. de Roode, "Positron extraction andtransport in a nuclear-reactor-based positron beam," Nuclear Instruments and Methods in Physics Research A, vol. 427, no. 1-2, pp. 266-270, 1999.

[5] A. W. Hunt, L. Pilant, D. B. Cassidy et al., "The development of the intense positron beam at Washington State University," Applied Surface Science, vol. 194, no. 1-4, pp. 296-300, 2002. 
[6] R. Krause-Rehberg, G. Brauer, M. Jungmann, A. Krille, A. Rogov, and K. Noack, "Progress of the intense positron beam project EPOS," Applied Surface Science, vol. 255, no. 1, pp. 2224, 2008.

[7] AIST, http://unit.aist.go.jp/riif/adcg/english.

[8] N. Oshima, R. Suzuki, T. Ohdaira et al., "A positron annihilation lifetime measurement system with an intense positron microbeam," Radiation Physics and Chemistry, vol. 78, no. 12, pp. 1096-1098, 2009.

[9] N. Oshima, R. Suzuki, T. Ohdaira et al., "Development of positron microbeam in AIST," Materials Science Forum, vol. 607, pp. 238-242, 2009.

[10] A. I. Hawari, D. W. Gidley, J. Xu et al., "The intense slow positron beam facility at the NC State University PULSTAR reactor," AIP Conference Proceedings, vol. 1099, pp. 862-865, 2009.

[11] A. I. Hawari, D. W. Gidley, J. Moxom, A. G. Hathaway, and S. Mukherjee, "Operation and testing of the PULSTAR reactor intense slow positron beam and PALS spectrometers," Journal of Physics: Conference Series, vol. 262, no. 1, Article ID 012024, 2011.

[12] C. Hugenschmidt, B. Löwe, J. Mayer et al., "Unprecedented intensity of a low-energy positron beam," Nuclear Instruments and Methods in Physics Research A, vol. 593, no. 3, pp. 616-618, 2008.

[13] S. Chemerisov and C. D. Jonah, "Development of high intensity source of thermal positrons APosS (Argonne Positron Source)," Journal of Physics: Conference Series, vol. 262, Article ID 012012, 2011.

[14] S. Golge, B. Vlahovic, and B. Wojtsekhowski, "High intensity low-energy positron source at Jefferson," in Proceedings of the International Particle Accelerator Conference (IPAC '12), 2012.

[15] D. B. Cassidy, S. H. M. Deng, R. G. Greaves, and A. P. Mills Jr., "Accumulator for the production of intense positron pulses," Review of Scientific Instruments, vol. 77, no. 7, Article ID 073106, 2006.

[16] N. Alberola, T. Anthonioz, A. Badertscher et al., "Development of a high-efficiency pulsed slow positron beam for measurements with orthopositronium in vacuum," Nuclear Instruments and Methods A, vol. 560, no. 2, pp. 224-232, 2006.

[17] D. J. Murtagh, "A positron buncher-cooler," The European Physical Journal D, vol. 68, p. 213, 2014.

[18] G. R. Brandes, K. F. Canter, T. N. Horsky, P. H. Lippel, and A. P. Mills Jr., "Scanning positron microbeam," Review of Scientific Instruments, vol. 59, no. 2, pp. 228-232, 1988.

[19] N. Oshima, R. Suzuki, T. Ohdaira et al., "Brightness enhancement method for a high-intensity positron beam produced by an electron accelerator," Journal of Applied Physics, vol. 103, no. 9, Article ID 094916, 2008.

[20] M. Fujinami, S. Jinno, M. Fukuzumi, T. Kawaguchi, K. Oguma, and T. Akahane, "Production of a positron microprobe using a transmission remoderator," Analytical Sciences, vol. 24, no. 1, pp. 73-79, 2008.

[21] D. B. Cassidy and S. Hogan, "Atom control and gravity measurements using Rydberg positronium," International Journal of Modern Physics: Conference Series, vol. 30, Article ID 1460259, 9 pages, 2014.

[22] P. Crivelli, U. Gendotti, A. Rubbia, L. Liszkay, P. Perez, and C. Corbel, "Measurement of the orthopositronium confinement energy in mesoporous thin films," Physical Review A, vol. 81, no. 5, Article ID 052703, 2010.
[23] D. B. Cassidy, P. Crivelli, T. H. Hisakado et al., "Positronium cooling in porous silica measured via Doppler spectroscopy," Physical Review A, vol. 81, no. 1, Article ID 012715, 12 pages, 2010.

[24] D. W. Gidley, H.-G. Peng, and R. S. Vallery, "Positron annihilation as a method to characterize porous materials," Annual Review of Materials Research, vol. 36, pp. 49-79, 2006.

[25] P. Crivelli, D. Cooke, B. Barbiellini et al., "Positronium emission spectra from self-assembled metal-organic frameworks," Physical Review B, vol. 89, Article ID 241103(R), 2014.

[26] D. B. Cassidy, T. H. Hisakado, V. E. Meligne, H. W. K. Tom, and A. P. Mills Jr., "Delayed emission of cold positronium from mesoporous materials," Physical Review A, vol. 82, Article ID 052511, 2010.

[27] D. B. Cassidy and A. P. Mills, "Enhanced Ps-Ps interactions due to quantum confinement," Physical Review Letters, vol. 107, Article ID 213401, 2011.

[28] S. D. Hogan and F. Merkt, "Demonstration of three-dimensional electrostatic trapping of state-selected rydberg atoms," Physical Review Letters, vol. 100, Article ID 043001, 2008.

[29] S. D. Hogan, C. Seiler, and F. Merkt, "Rydberg-state-enabled deceleration and trapping of cold molecules," Physical Review Letters, vol. 103, Article ID 123001, 2009.

[30] S. Mariazzi, P. Bettotti, and R. S. Brusa, "Positronium cooling and emission in vacuum from nanochannels at cryogenic temperature," Physical Review Letters, vol. 104, Article ID 243401, 2010.

[31] D. B. Cassidy, T. H. Hisakado, H. W. K. Tom, and A. P. Mills, "Optical spectroscopy of molecular positronium," Physical Review Letters, vol. 108, no. 13, Article ID 133402, 2012.

[32] G. Consolati, R. Ferragut, A. Galarneau, F. di Renzo, and F. Quasso, "Mesoporous materials for antihydrogen production," Chemical Society Reviews, vol. 42, no. 9, pp. 3821-3832, 2013.

[33] A. P. Mills, "Positronium formation at surfaces," Physical Review Letters, vol. 41, article 1828, 1978.

[34] M. S. Fee, A. P. Mills Jr., S. Chu et al., "Measurement of the positronium 13S1-23 S1 interval by continuous-wave twophoton excitation," Physical Review Letters, vol. 70, no. 10, pp. 1397-1400, 1993.

[35] K. P. Ziock, R. H. Howell, F. Magnotta, R. A. Failor, and K. M. Jones, "First observation of resonant excitation of high-n states in positronium," Physical Review Letters, vol. 64, no. 20, pp. 2366-2369, 1990.

[36] D. B. Cassidy, T. H. Hisakado, H. W. K. Tom, and A. P. Mills, "Optical spectroscopy of molecular positronium," Physical Review Letters, vol. 108, Article ID 133402, 2012.

[37] A. C. L. Jones, T. H. Hisakado, H. J. Goldman, H. W. K. Tom, A. P. Mills Jr., and D. B. Cassidy, "Doppler-corrected Balmer spectroscopy of Rydberg positronium," Physical Review A, vol. 90, Article ID 012503, 2014.

[38] P. Crivelli, D. A. Cooke, and S. Friedreich, "Experimental considerations for testing antimatter antigravity using positronium 1S-2S spectroscopy," International Journal of Modern Physics: Conference Series, vol. 30, Article ID 1460257, 10 pages, 2014.

[39] G. Dufour, A. Gerardin, R. Guerout et al., "Quantum reflection of antihydrogen from the Casimir potential above matter slabs," Physical Review A, vol. 87, Article ID 012901, 2013.

[40] A. Badertscher, P. Crivelli, W. Fetscher et al., "Improved limit on invisible decays of positronium," Physical Review D, vol. 75, no. 3, Article ID 032004, 2007.

[41] T. F. Gallagher, Rydberg Atoms, Cambridge University Press, Cambridge, Mass, USA, 1994. 
[42] A. Kellerbauer, M. Amoretti, A. S. Belov et al., "Proposed antimatter gravity measurement with an antihydrogen beam," Nuclear Instruments and Methods in Physics Research B, vol. 266, pp. 351-356, 2008.

[43] D. P. van der Werf and GBAR collaboration, "The GBAR experiment," International Journal of Modern Physics: Conference Series, vol. 30, Article ID 1460263, 2014.

[44] A. I. Zhmoginov, A. E. Charman, R. Shalloo, J. Fajans, and J. S. Wurtele, "Nonlinear dynamics of anti-hydrogen in magnetostatic traps: implications for gravitational measurements," Classical and Quantum Gravity, vol. 30, no. 20, Article ID 205014, 2013.

[45] P. J. Mohr, B. N. Taylor, and D. B. Newell, "CODATA recommended values of the fundamental physical constants: 2010," Reviews of Modern Physics, vol. 84, article 1527, 2012.

[46] R. Pohl, A. Antognini, F. Nez et al., "The size of the proton," Nature, vol. 466, pp. 213-216, 2010.

[47] A. Antognini, F. Nez, K. Schuhmann et al., "Proton structure from the measurement of $2 \mathrm{~S}-2 \mathrm{P}$ transition frequencies of muonic hydrogen," Science, vol. 339, no. 6118, pp. 417-420, 2013.

[48] F. Nez, A. Antognini, F. D. Amaro et al., "Is the proton radius a player in the redefinition of the international system of units?" Philosophical Transactions of the Royal Society A, vol. 369, no. 1953, pp. 4064-4077, 2011. 

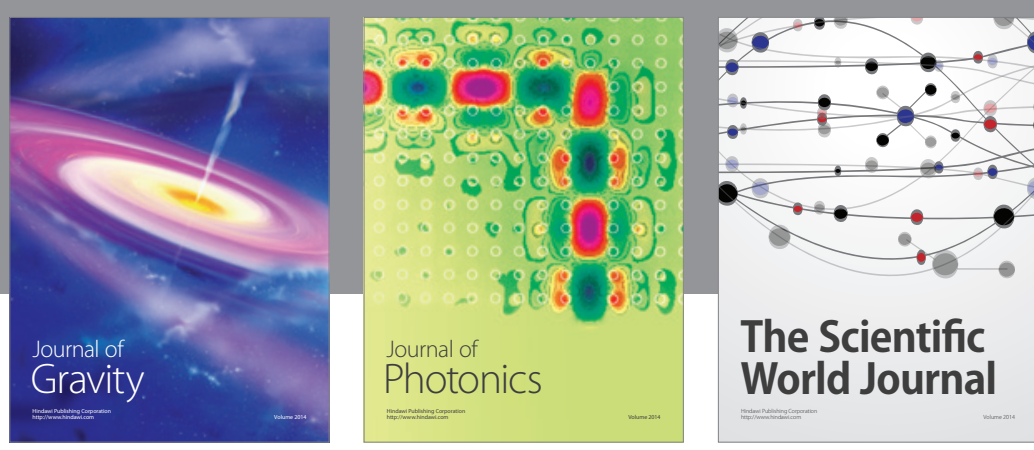

The Scientific World Journal
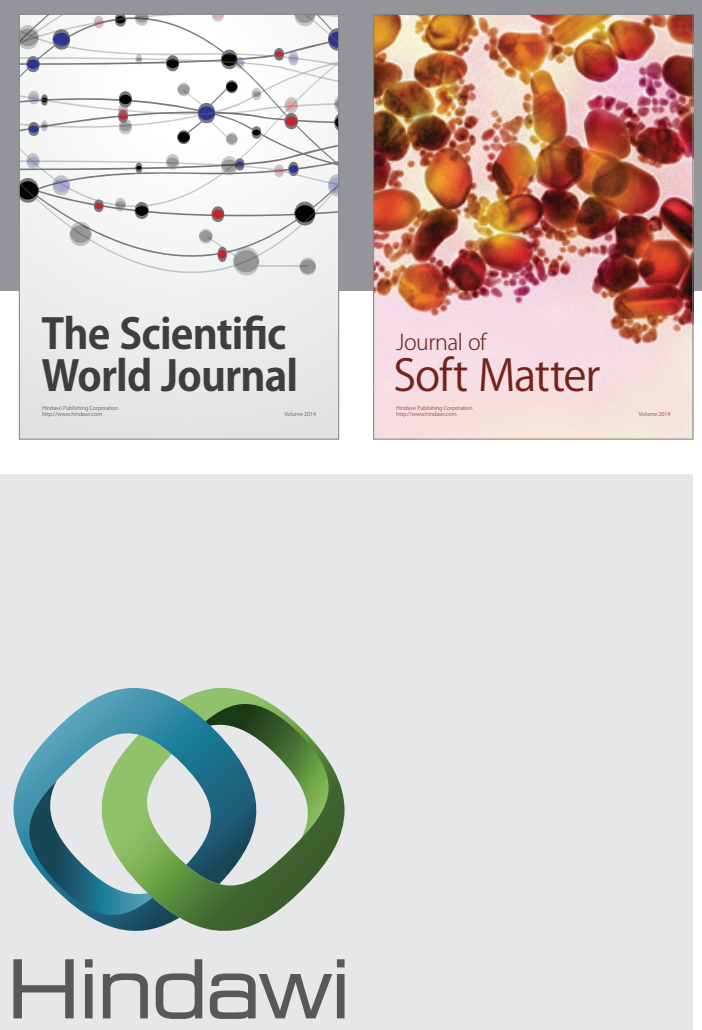

Submit your manuscripts at

http://www.hindawi.com

nternational Journal of

Statistical Mechanics
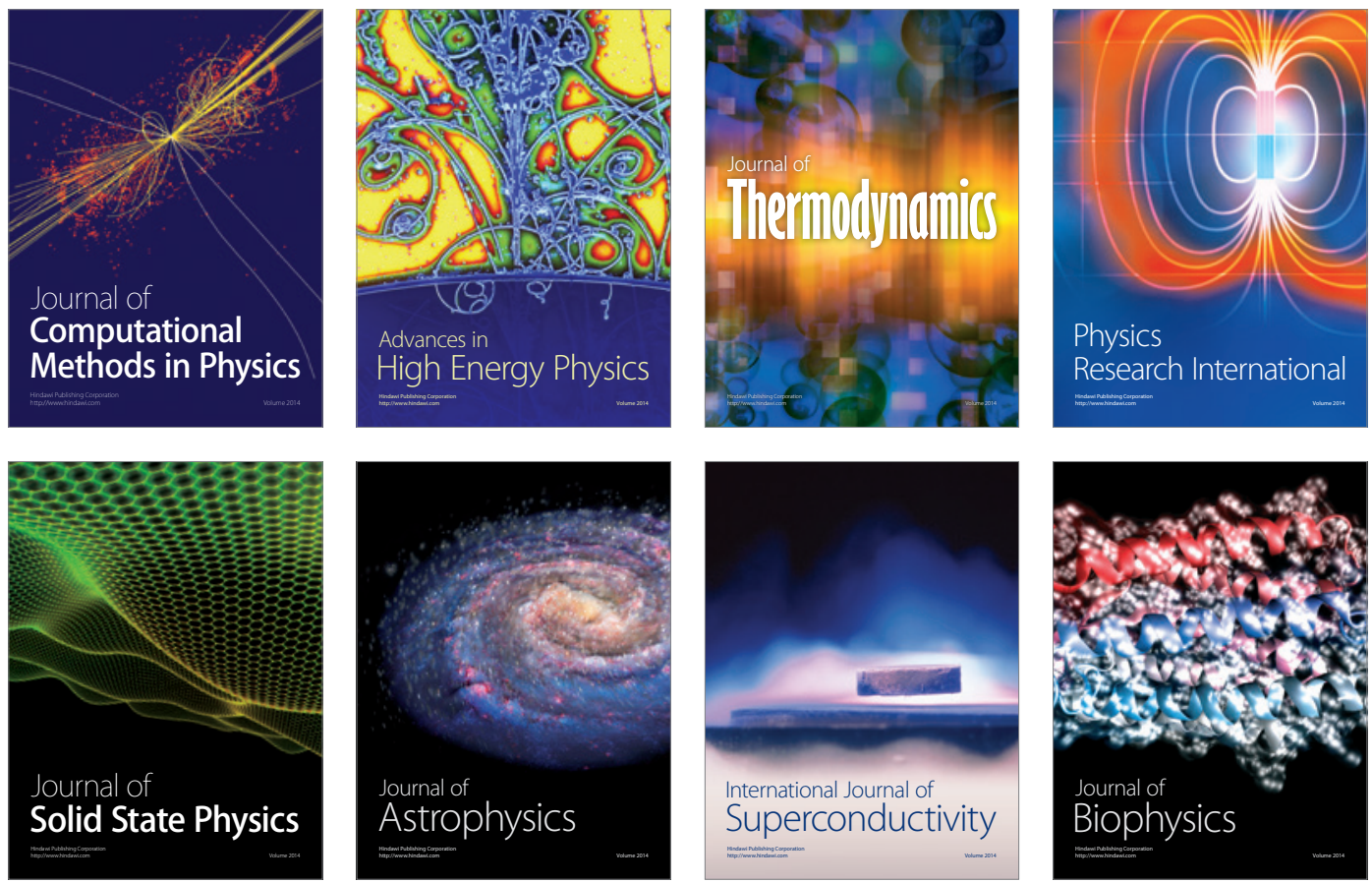
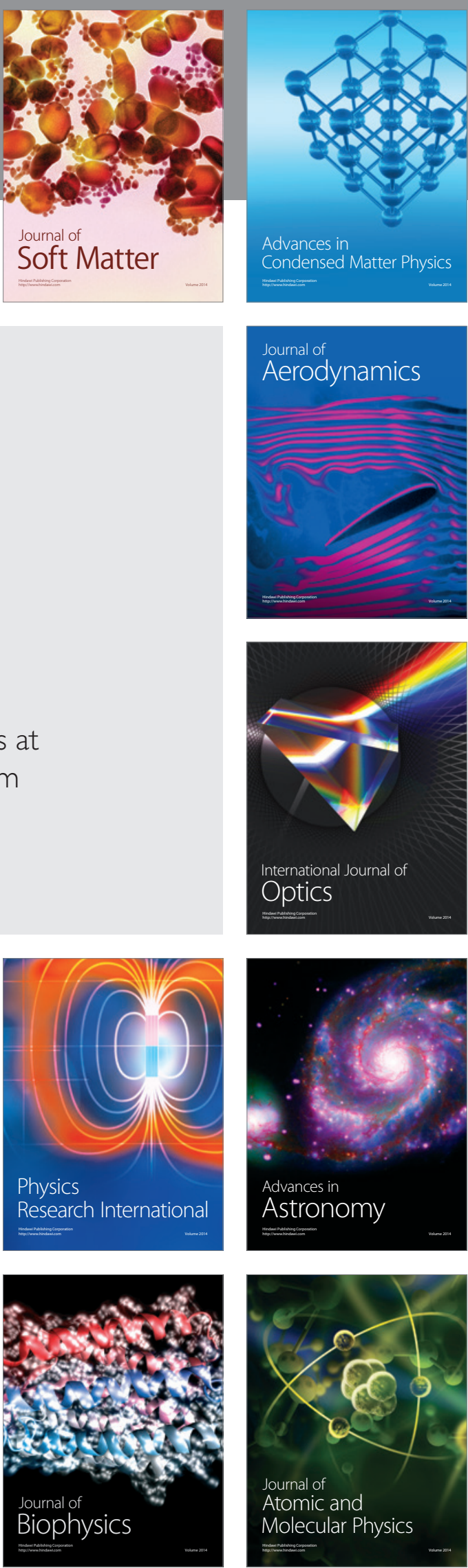